\title{
Interaction Force between a Small Magnet and a Superconducting Sphere
}

\author{
Ahmad Subahi ${ }^{1}$ and Mutazz Nuseirat ${ }^{2}$ \\ ${ }^{1}$ Department of Basic Sciences, College of Science and Health Professions, King Saud Bin Abdulaziz \\ University for Health Sciences, Mail Code 6666 P.O.Box 9519, Jeddah 21423, and ${ }^{2}$ Department of \\ Basic Sciences, College of Science and Health Professions, King Saud Bin Abdulaziz University for \\ Health Sciences, Mail Code 3124, P.O.Box 22490, Riyadh 11426, Saudi Arabia
}

Subahia@ksau-hs.edu.sa

\begin{abstract}
The interaction force between a small magnet and a superconducting sphere is calculated using the method of images. Our approach can be easily verified by applying boundary conditions. This work will possesses and clear the discrepancies found in the literature. For collinear dipoles case (small magnet and its image), we found the elevated force to be dependent on the geometrical dimensions of the problem.
\end{abstract}

Keywords: Levitation force, Image method, Meissner state, Magnetic field.

\section{Introduction}

Magnetic levitation (maglev) is the concept of suspending objects with no support but magnetic field. Maglev is used for magnetic applications, transportation and product display purposes. The problem of an elevated magnet above a superconducting material has been investigated by many researchers ${ }^{[1,2,3]}$. The case of a small magnet elevated above a semi-infinite superconducting material has no discrepancy in literature regardless of the method used to estimate the interaction or the elevated force between the magnet and the semi-infinite superconducting material. This is because the radius of curvature is infinite and the boundary conditions at the semiinfinite plane can be easily verified ${ }^{[4,5]}$. However, in the cases of curved surfaces, applying the boundary conditions are more difficult. Therefore, those who worked with curved surfaces, such as spherical surfaces, came up with different results ${ }^{[6,7,8]}$. When we follow their derivations we found that their results did not match the boundary condition that such surfaces possess ${ }^{[6,7]}$. Some authors when they generalize the problem they fixed the image position and calculated the magnetic field at the position of the magnet [8], and they ignored the fact that the magnet and its image are collinear and you cannot fix one and ignore the other.

In this work the interaction force between a small magnet and a superconducting sphere had been calculated using the method of images [9]. We will demonstrate our method of calculations in a simple manner that can be easily followed and the boundary conditions can be verified.

\section{Theory}

The problem introduced in this work consists of a small magnet elevated above a superconducting sphere of radius $\mathrm{a}$ in the 
Meissner state. The small magnet will be replaced by a dipole source $(\vec{m})$ pointed out in the direction of $\vec{r}$ as shown in Fig. 1. This assumption can be justified by assuming that the radius of the sphere is so large compared to the dimensions of the tiny magnet. The presence of the magnet will produce a surface super current density on the surface of the sphere. The penetration depth will be ignored since the Meissner state has been established by the magnetic field of the small magnet and no magnetic field penetrates the interior of the sphere. The frame of reference will be at the center of the sphere and the magnet (source) will be placed outside the sphere at position (z) above the center of the sphere where, $\mathrm{z}>$ a. The magnetic dipole which represents the small magnet is directed radially out relative to our system of coordinates. Now, applying the Image method concepts ${ }^{[9]}$, the image of the magnet must be located inside the sphere and its magnetization points toward the center of the sphere. The image is located at distance (b) from the center of the sphere and its collinear with source. In order to figure out the location of the image, we must apply the boundary condition at the surface of the sphere. The magnetic field $\vec{B}$ has no normal component at the surface of the sphere.

Now, let us calculate the magnetic field due to the source and its image in the physical region, which is outside the sphere. The magnetic field $\vec{B}$ due to a magnetic dipole is given by:

$$
\vec{B}_{\text {dipole }}=\frac{\mu_{o}}{4 \pi r^{3}}((3 \vec{m} \cdot \hat{r}) \hat{r}-\vec{m})
$$

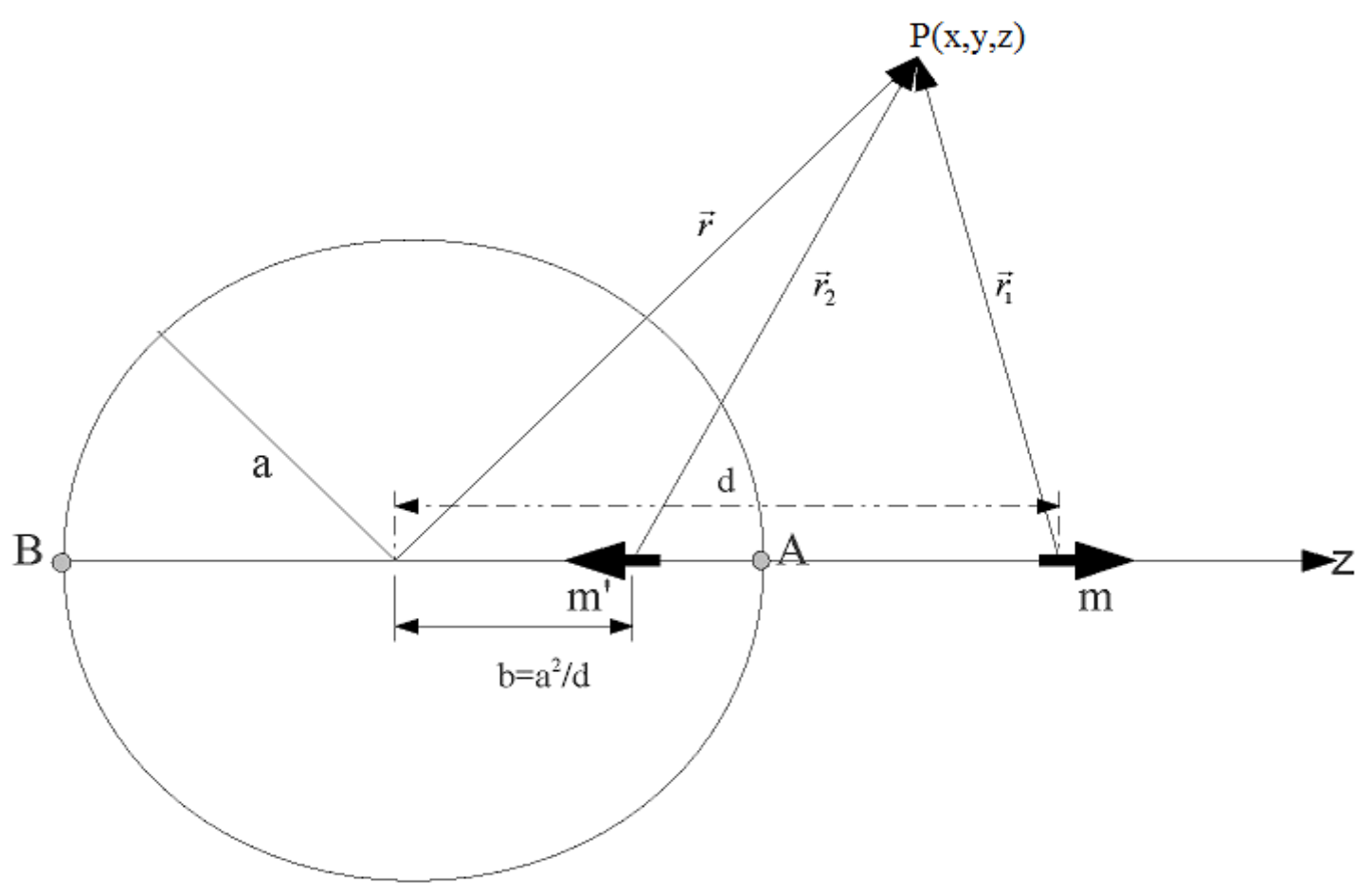

Fig. 1. Schematic illustration of the superconducting sphere-dipole system. 
We will assume that the source (small magnet) has a magnetic dipole moment $\vec{m}$ and its image $\vec{m}^{\prime}$. Note $\vec{m}^{\prime}$ is opposite in direction to $\vec{m}$ as seen in Fig. 1 . The total magnetic field at arbitrary point $\mathrm{P}(\mathrm{x}, \mathrm{y}, \mathrm{z})$ outside the sphere is due the vector sum of the dipole moment and its image, and its given by:

$$
\vec{B}=\frac{\mu_{o}}{4 \pi r_{1}^{3}}\left(\left(3 \vec{m} \cdot \hat{r}_{1}\right) \hat{r}_{1}-\vec{m}\right)+\frac{\mu_{o}}{4 \pi r_{2}^{3}}\left(\left(3 \vec{m}^{\prime} \cdot \hat{r}_{2}\right) \hat{r}_{2}-\vec{m}^{\prime}\right)
$$

Where $\vec{r}_{1}$ and $\vec{r}_{2}$ are the distances from $\vec{m}$ and $\vec{m}^{\prime}$ to the point $\mathrm{P}(\mathrm{x}, \mathrm{y}, \mathrm{z})$ respectively. The first step in our calculations is to derive the values of the image dipole $\vec{m}^{\prime}$ and its location $\mathrm{b}$ in terms of $\vec{m}, a$, and $d$, which are the known quantities in our problem. This can be achieved by applying the boundary condition at the surface of the sphere, where the normal component of the magnetic field $\vec{B}$ is equal to zero. We will take two special points (A) and (B) at the surface of the sphere as shown in Fig. 1.

Then, at point (A) equation (1) reduces to:

$$
\frac{2 \mu_{0} m}{4 \pi(d-a)^{3}}-\frac{2 \mu_{0} m^{\prime}}{4 \pi(a-b)^{3}}=0
$$

And at point (B) we have:

$$
\frac{2 \mu_{0} m}{4 \pi(d+a)^{3}}-\frac{2 \mu_{0} m^{\prime}}{4 \pi(a+b)^{3}}=0
$$

Solving (2) and (3) for $b$ and $m^{\prime}$ we obtain:

$$
\vec{m}^{\prime}=-\left(\frac{a}{d}\right)^{3} \vec{m} \text { and } b=\frac{a^{2}}{d}
$$

These two expressions assure that the normal magnetic field $\mathrm{B}$ at the surface of the sphere is zero. This can be verified by substituting for $\vec{m}^{\prime}$ and $b$ back in equation (1) after extracting the normal component $\vec{B}$ in any direction normal to the surface of the sphere.
Now to calculate the interaction force between the source (small magnet) and the sphere we will simply calculate the force on the source due to field of the image dipole $\left(\vec{B}^{\prime}\right)$ at position $z$, where the small magnet is located and noticing that the image and the source lay at the same line. So, the force is given by $\vec{F}=\vec{\nabla}\left(\vec{m} \cdot \vec{B}^{\prime}\right)$.

Since,

$\vec{m} \cdot \vec{B}^{\prime}=\vec{m} \cdot\left(-\frac{2 \mu_{o} m(a / z)^{3} \hat{k}}{4 \pi\left(z-a^{2} / z\right)^{3}}\right)=\vec{m} \cdot\left(-\frac{2 \mu_{o} m a^{3} \hat{k}}{4 \pi\left(z^{2}-a^{2}\right)^{3}}\right)=-\frac{2 \mu_{o} m^{2} a^{3}}{4 \pi\left(z^{2}-a^{2}\right)^{3}}$

Therefore, the force is

$$
\vec{F}=\frac{\partial}{\partial z}\left(\frac{-2 \mu_{o} m^{2} a^{3}}{4 \pi\left(z^{2}-a^{2}\right)^{3}} \hat{k}\right)=\frac{6 \mu_{o} m^{2} a^{3} z}{2 \pi\left(z^{2}-a^{2}\right)^{4}} \hat{k}
$$

If the distance between the source and the center of the sphere $d$ then the force is equal to:

$$
F=2 \frac{3 \mu_{o} m^{2} a^{3} d}{2 \pi\left(d^{2}-a^{2}\right)^{4}}
$$

\section{Results and Discussion}

In fact the force given in equation (6) is twice of that given in Ref. [8] due to the fact that the generalization adopted by the author in Ref. [8] (equation (10) in particular in Ref. [8]) did fix the position of the image and then deduce the derivative.

$$
F=\frac{3 \mu_{0} m^{2}}{2 \pi} \frac{a^{3} d}{\left(d^{2}-a^{2}\right)^{4}}
$$

This is not mathematically correct because the image and the source lay in the same line and connected together by equation (4). In other words if the magnet moves a way along the z-axis, the image will move in the opposite direction toward the origin and vice versa. However, in the above mentioned reference, the authors did fix the position of the image while the position of the magnet kept varying. Moreover, the magnetic potential 
energy calculated here is also twice of what has been calculated in Ref. [8]. This can be seen in our calculations in equation (5) that represents the magnetic potential energy stored in the system. This is because the sphere is presented in the external field of the small magnet and the factor half should not appear. In fact the magnet is an external agent of magnetic field, where the superconducting sphere located.

\section{Conclusions}

In this work we have calculated the levitation force on a small magnet in the presence of a superconductor sphere in Meissner state, using the method of Images. We have cleared the discrepancy founded in literatures. Our calculations are very clear and can be easily traced.

\section{Acknowledgement}

We would like to thank Professor M.K. Qaseer at Jordan University of Science and Technology for his role in the accomplishment of this research paper.

\section{References}

[1] Alqadi, M.K., Alzoubi, F. Y., Al-Khateeb, H. M., Saasdeh, S. M. and Ayoub, N. Y. (2012), "Force analysis of a permanent magnet and superconducting hollow cylinder", J. Supercond Nov Magn, 25(1): 1469-73.

[2] Al-Khateeb, H.M., Alqadi, M.K., Alzoubi, F.Y., Albiss, B., Hasan (Qaseer), M.K. and Ayoub., N.Y. (2016), "Levitation and lateral forces between a point magnetic dipole and a superconducting sphere", Chinese Physics B, 25(5): 58402.

[3] Alzoubi, F.Y., Alqadi, M. K., Al-Khateeb, H. M. and Ayoub, N.Y. (2005) "Size Effects on Levitation Force Between a Magnet and a Superconducting Thin Film in the Meissner State", Supercond. Sci. Technol, 18: 227230 .

[4] Lugo. J. and Sosa, V. (1999), "Levitation force between a small magnet and a superconducting sample of finite size in the Meissner state", Physica C 324: 9-14.

[5] Diaz, J.L.P and Prada, J.C.G. (2007), "Mechanical Method for Experimental Determination of the FirstPenetration Field in High-Temperature Superconductors", Physica C 467: 141.

[6] Coffey., Mark W. (2000), "Levitation Force Between a Point Magnetic Dipole and Superconducting Sphere", Journal of Superconductivity, 13(3): 381-388.

[7] Palaniappan, D. (2009), "Magnetic Interaction Force and a Couple on a Superconducting Sphere in an Arbitrary Dipole Field", J. Supercond, 22(5): 471-477.

[8] Lin, Qiong-Gui (2006), "Theoretical development of the image method for a general magnetic source in the presence of a superconducting sphere or a long superconducting cylinder", Phys. Rev. B, 74: 024510.

[9] Jackson, J.D. (1999), Classical Electrodynamics, 3rd Edition, Wiely. 


\section{قوة التفاعل بين المغناطيس الصغير والمجال الكروي فائق التوصيل}

\section{أحمد سبحي' و معتز نصيرات}

' قسم العلوم الأساسية، كلية العلوم والمهن الصحية، جامعة الملك سعود بن عبدالعزيز للعلوم الصحية - جدة. و قَسم العلوم

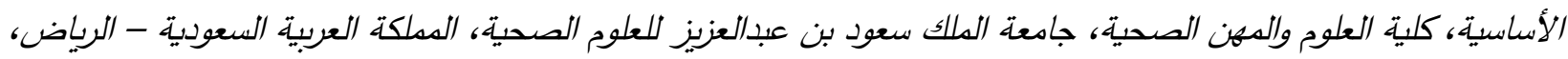

$$
\text { المدلكة العربية السعودية }
$$

subahia@ksau-hs.edu.sa

المستخلص. تم احتساب قوة التقاعل بين المغناطيس الصغير والمجال فائق التوصيل الكروي باستخدام طريقة الصور • يمكن التحقق من منهجنا في العمل بسهولة من خلال الظروف الحدودية وبيان التناقضات الموجودة في الدراسات السابقة، بالنسبة لحالة الثناقطب المغناطيسي الصغير المتسق خطيًا وصورته، وجد أن قوة الرفع تعتمد على الأبعاد الهندسية للمشكلة. كلمات مفتاحية: قوة الرفع، طريقة الصورة، حالة ميسنر ، المجال المغناطيسي. 
